\section{A) Check for updates}

Cite this: Nanoscale, 2021, 13, 3746

\title{
Femtosecond laser driven precessing magnetic gratings $\uparrow$
}

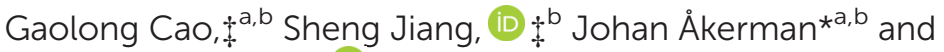 \\ Jonas Weissenrieder (iD) *a
}

\begin{abstract}
Manipulation and detection of spins at the nanoscale is of considerable contemporary interest as it may not only facilitate a description of fundamental physical processes but also plays a critical role in the development of spintronic devices. Here, we describe the application of a novel combination of transient grating excitation with Lorentz ultrafast electron microscopy to control and detect magnetization dynamics with combined nanometer and picosecond resolutions. Excitation of $\mathrm{Ni}_{80} \mathrm{Fe}_{20}$ thin film samples results in the formation of transient coherently precessing magnetic gratings. From the time-resolved results, we extract detailed real space information of the magnetic precession, including local magnetization, precession frequency, and relevant decay factors. The Lorentz contrast of the dynamics is sensitive to the alignment of the in-plane components of the applied field. The experimental results are rationalized by a model considering local demagnetization and the phase of the precessing magnetic moments. We envision that this technique can be extended to the study of spin waves and dynamic behavior in ferrimagnetic and antiferromagnetic systems.
\end{abstract}

Received 7th November 2020

Accepted 17th January 2021

DOI: 10.1039/d0nr07962f

rsc.li/nanoscale distributions,${ }^{16}$ TGS can be predicted to form a sensitive probe of ultrafast magnetization dynamics. A few pioneering works have studied TG-induced magnetic excitation of surface magnetoelastic waves, ${ }^{16}$ spin currents ${ }^{17}$ and writing of strip domains. ${ }^{18}$ However, TG-induced ultrafast dynamics of magnetic systems have been sparsely investigated at large, although it may be expected to provide rich physics and hold potential applications within e.g. spintronics. ${ }^{19,20}$ This may be traced to the fact that TGS has been mostly restricted to timeresolved spectroscopy, leaving real space information mostly unknown. Combined temporal and spatial information are important for the understanding of spintronic process as the TG period acts on length scales of relevance to intrinsic magnetic properties, such as the exchange coupling and spin diffusion length scales. Application of electron beam probes, at inherent high spatial resolution, may circumvent the spatial resolution restrictions of optical probes imposed from diffraction limitations. Commercial transmission electron microscopy (TEM), in Lorentz mode, routinely demonstrates nanometer resolution for magnetic structures. ${ }^{21}$ The recent developments within Lorentz ultrafast TEM (LUEM), through integration of pump-probe into the system, ${ }^{22-24}$ have demonstrated its ability for studies of ultrafast magnetization dynamics on systems such as skyrmions, vortices, and domain walls. ${ }^{25-29}$ Direct imaging of magnetization dynamics at combined nanoscale and picosecond resolutions may uncover new details central to the understanding of spintronic processes including ultrafast magnetic switching, formation and pertur-

\footnotetext{
${ }^{a}$ Materials and Nano Physics, Department of Applied Physics, KTH Royal Institute of Technology, Kista, Sweden. E-mail: jonas@kth.se

${ }^{b}$ Department of Physics, University of Gothenburg, Gothenburg, Sweden. E-mail: johan.akerman@physics.gu.se

$\dagger$ Electronic supplementary information (ESI) available: Materials and methods; sections S1-S6. See DOI: 10.1039/d0nr07962f

$\$$ These authors contributed equally to this work.
} 
bation of topological magnetic structures, and spin wave propagation.

Motivated by the above, we set out to develop a technique combining TG with LUEM. A fs UV laser pulse and a visible TG were established in a modified TEM. The TG-induced spin dynamics was probed by a photoemitted electron pulse. Specifically, we use an optical TG to excite a ferromagnetic sample, $\mathrm{Ni}_{80} \mathrm{Fe}_{20}$, deposited on a $\mathrm{Si}_{3} \mathrm{~N}_{4}$ membrane. The consequence of the TG excitation is a spatially periodic laser-driven demagnetization that is coupled to a subsequent formation of temperature gradients on the nm scale. The magnetic response of the sample results in a periodic Lorentz image contrast. Time-resolved analysis of oscillations in the local Lorentz contrast, under the influence of an external magnetic field, allows for the determination of the phase and frequency of local precessing magnetic moments, local magnetization, and relevant decay factors of the magnetic grating, which is a collective coherent in-phase precession of magnetization with a spatially periodic modulation of the amplitude. The presented results demonstrate that the technique is capable of investigating spin dynamics at combined ps temporal and nm spatial resolutions. The application of TG-induced magnetic gratings may tentatively be extended to studies of spin waves as the analysis is sensitive to the phase of the precession and may be deployed under external fields. The technique also holds promise for application in other magnetic systems, such as ferri- or antiferromagnets.

\section{Results}

Fig. 1 shows the schematic illustration of the LUEM setup with a transient grating on a magnetic sample. Unlike in conventional LUEM pump-probe schemes, which employ a single spatially homogeneous fs pump laser to initiate dynamics, two fs pump beams (at $\lambda=515 \mathrm{~nm}$ or $1030 \mathrm{~nm}$ ) are deployed to form the TG on the sample. Two colors of the spins are used to illustrate the regions of constructive and destructive interference on the sample, where red represents high local laser fluence and blue low local laser fluence. The periodic excitation results in formation of coupled thermal and magnetic gratings. A photoemitted electron probe pulse, arriving at an adjustable time delay, detects the local magnetization of the sample. The photoinduced demagnetization of the sample is responsible for the Lorentz contrast and offers a means to study the laser-induced magnetization dynamics.

Fig. 2A shows the schematic illustration of the experimental geometry for a ferromagnetic sample placed on a double-tilt holder in the LUEM. The $\mathrm{Ni}_{80} \mathrm{Fe}_{20}$ film is deposited on a thin $\mathrm{Si}_{3} \mathrm{~N}_{4}$ membrane with slanted faces surrounding the sample surface. The pump laser beam is carefully aligned to illuminate both one slanted face and the membrane. The laser pulse reflected from the slanted face interferes with the direct laser pulse at the membrane surface, resulting in an optical grating with a periodic distribution of the laser intensity. An external magnetic field may be applied along the $Z$ direction and the

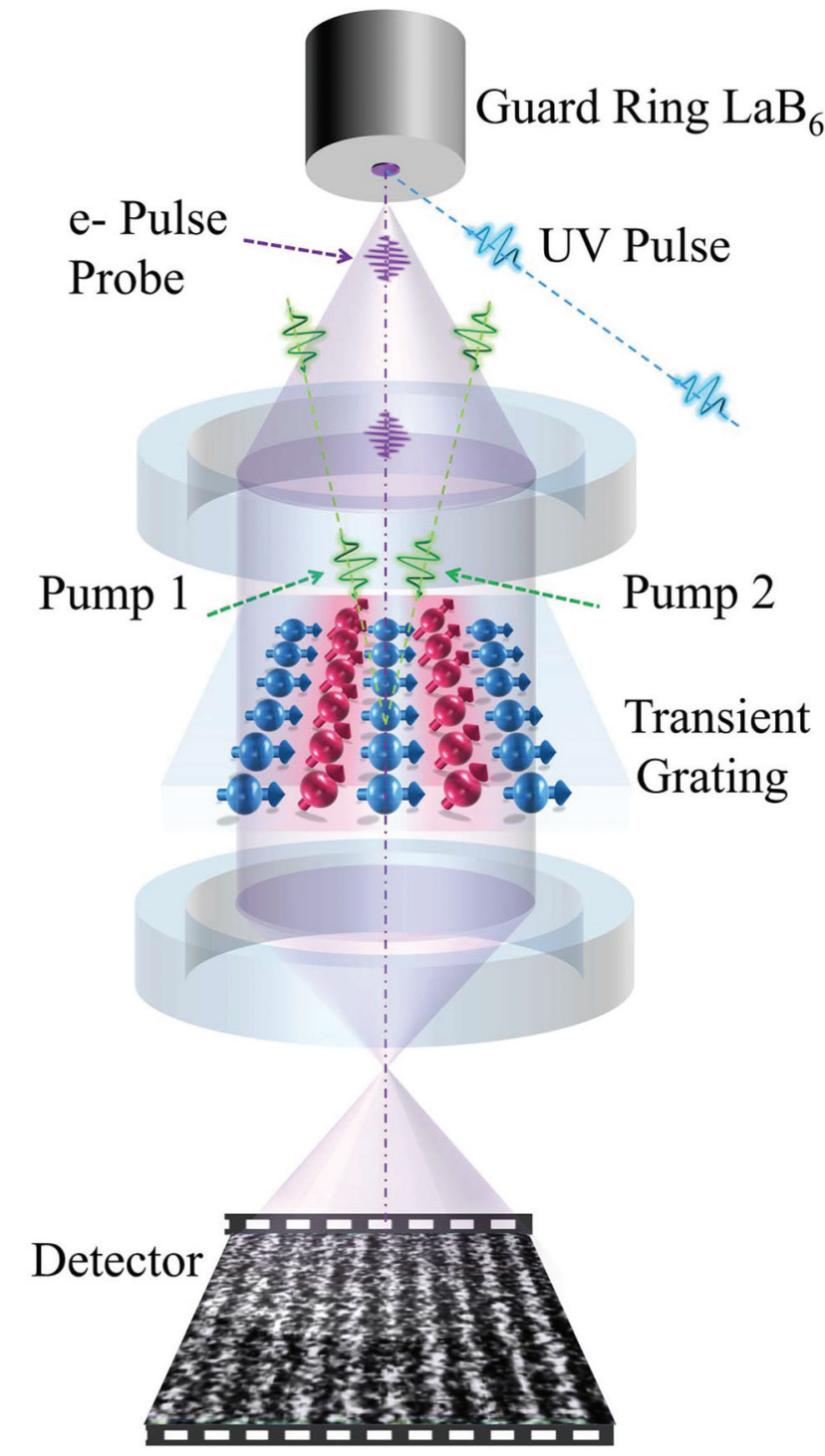

Fig. 1 Schematic illustration of time-resolved imaging of magnetization dynamics using LUEM with transient grating excitation. The two optical pulses (pump 1 and pump 2) interfere at the sample position to form a transient grating. The magnetic response of the sample is probed by an electron pulse at variable delay and recorded as Lorentz contrast at the detector.

in-plane component of the field can be controlled by applying sample tilt angles $\alpha$ and $\beta$. Excitation with this TG then results, through electron-phonon coupling, in a transient thermal grating. The optical excitation process and the following transient thermal grating in turn result in a patterned ultrafast demagnetization of the permalloy sample with the same periodicity as the TG. Inset of Fig. 2B shows the LUEM images in Fresnel mode at an over-focus of $5 \mathrm{~mm}$ collected before $\left(-25 \mathrm{ps}, T_{0}\right)$ and after $\left(+175 \mathrm{ps}, T_{2}\right)$ excitation with a femtosecond laser pulse at a fluence of $9 \mathrm{~mJ} \mathrm{~cm}^{-2}$. The Lorentz image collected before excitation, at $-25 \mathrm{ps}$, shows no magnetic contrast, while the image collected +175 ps after excitation exhibits periodic stripes of magnetic contrast. The striped magnetic contrast is formed parallel to the slanted face 

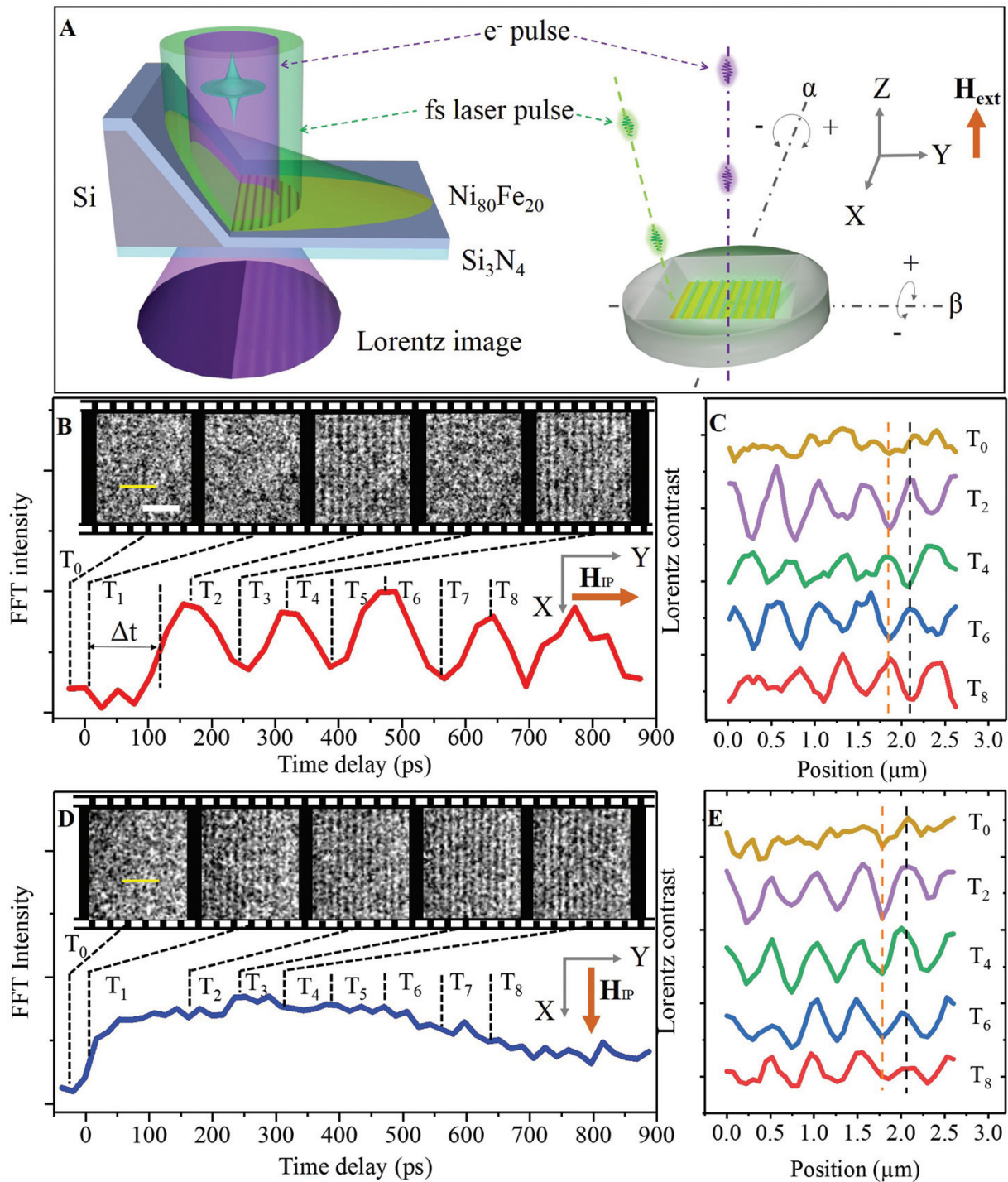

Fig. 2 Schematic illustration of the details of experimental setup and temporal evolution of the periodic magnetic contrast. A magnetic sample ( $50 \mathrm{~nm}$ permalloy, $\mathrm{Ni}_{80} \mathrm{Fe}_{20}$ ) is deposited on a thin $\mathrm{Si}_{3} \mathrm{~N}_{4}$ membrane $(50 \mathrm{~nm}$ ). The transient optical grating is formed from interference between the direct pump beam and the beam reflected from the slanted face of the membrane. The sample is placed on a double tilt holder that allows precise adjustment of tilt angles $\alpha$ and $\beta$, which allows control of the in-plane component $\left(H_{\mathrm{IP}}\right)$ of the external magnetic field $H_{\text {ext. }}$ (B) Selected time-resolved Lorentz images and the corresponding temporal trace of the FFT intensity of the magnetic contrast at an external magnetic field of $\mu_{0} H_{\text {ext }}=250 \mathrm{mT}$. The in-plane component of the magnetic field, $\boldsymbol{H}_{\mathrm{IP}}$, is oriented along the $Y$ axis by applying tilt angles of $\alpha=3^{\circ}$ and $\beta=0^{\circ}$. A series of images are purposely selected to show an oscillation in magnetic contrast. $T_{2}, T_{4}, T_{6}$ and $T_{8}$ are the images collected at maximum magnetic contrast and $T_{1}, T_{3}, T_{5}$ and $T_{7}$ are the images taken at minimum magnetic contrast. The scale bar (white solid line) indicates $2 \mu \mathrm{m}$. (C) Selected line profiles, at the dashed yellow line in (B), at time delays $T_{0}, T_{2}, T_{4}, T_{6}$ and $T_{8}$. (D) Selected time-resolved Lorentz images and the corresponding FFT intensity temporal trace at a magnetic field of $\mu_{0} H_{\text {ext }}=250 \mathrm{mT}$ and with $H_{\mathrm{IP}}$ oriented along the $X$ axis $\left(\alpha=0^{\circ}\right.$ and $\left.\beta=3^{\circ}\right)$. $\Delta t$ in (B) indicates the temporal difference between the inflection points of the rising edges in (B) and (D). (E) Selected line profiles (at the dashed the yellow line in (D)) at time delays $T_{0}, T_{2}, T_{4}, T_{6}$ and $T_{8}$ in (D).

of the transmission electron microscopy membrane grid. We note that the periodic stripes show no magnetic contrast when the sample is placed in Fresnel mode focus conditions and that the contrast is reversed in under-focus conditions (section S1†). These observations confirm that the periodic structure is indeed a result of magnetic Lorentz contrast. In order to deduce the relationship between the periodic magnetic contrast and the TG (interference pattern), we conducted similar measurements at varying sample tilt angles, $\alpha$. The periodicity of the magnetic contrast, $d$, increases with increasing $\alpha$. The periodicity of the laser interference pattern $d$ in our local experimental geometry can be expressed as (see section $\mathrm{S} 2 \dagger$ ):

$$
d=\frac{\frac{\lambda \cdot \sin (2 \varphi+\alpha) \cos (\alpha)}{1-\cos (2 \varphi+2 \alpha)}}{1+\frac{\cos (2 \varphi+\alpha) \sin (\varphi)}{\sin (\varphi+\alpha)}}
$$


where $\varphi=35^{\circ}$ is the angle of the slanted face with respect to the normal of the sample surface, $\lambda$ is the wavelength of the laser (515 or $1030 \mathrm{~nm}$ ), and $\alpha$ is the tilt angle of the sample along the $Y$ axis shown in Fig. 2A. The calculated periodicity of the optical grating $d$ is in excellent agreement with the experimental results from the periodic magnetic contrast (see Fig. S2 (B) $\dagger$ ). This is further corroborated by the observation that when doubling the laser wavelength $\lambda$ from $515 \mathrm{~nm}$ to $1030 \mathrm{~nm}$, the periodicity of the magnetic contrast is increased by a factor of two (see section $\mathrm{S} 3 \dagger$ ). These results lead us to conclude that a TG can be used for writing a transient periodic magnetic structure in permalloy and the periodicity of the magnetic structure can be precisely tuned by changing the interference conditions. $^{30-32}$

We now turn our attention to the temporal evolution of the photo-induced transient magnetic grating. Fig. 2B shows the temporal trace of the FFT intensity of the periodic magnetic contrast and Lorentz images collected at selected delay times. The results were obtained under an external magnetic field of $\mu_{0} H_{\text {ext }}=250 \mathrm{mT}$ with the in-plane component $\boldsymbol{H}_{\mathbf{I P}}$ oriented along the $Y$ axis (see Fig. 2A) of the sample (sample tilt angles: $\alpha=3^{\circ}$ and $\beta=0^{\circ}$ ). A gradual increase in the periodic magnetic contrast is observed following laser excitation (after time zero) with a first maximum reached at approximately $175 \mathrm{ps}\left(T_{2}\right.$ in Fig. 2B). At longer delays, the FFT intensity oscillates with a decay on the nanosecond time scale. Fig. $2 \mathrm{~B}$ also presents the representative Lorentz UEM images collected at temporal delays, where the FFT intensity exhibits local maxima and minima. Fig. 2C shows the line profiles extracted from the Lorentz images in Fig. $2 \mathrm{~B}$ at the position indicated by the dashed yellow line. The line profiles were extracted at a temporal delay before time zero $\left(T_{0}\right)$ and at delays corresponding to the first four maxima of the FFT intensity $\left(T_{2}, T_{4}, T_{6}\right.$ and $T_{8}$ ). A careful inspection of the phase of the line profiles reveals that neighboring (in time) FFT maxima exhibit opposite magnetic contrast at a given spatial position. The contrast reversal can be observed by following the red and black vertical dashed lines in Fig. 2C and implies that the oscillation in the FFT intensity represents a periodic reversal of the Lorentz contrast from the magnetic structure. The line profile before time zero ( $T_{0}$, Fig. $\left.2 \mathrm{C}\right)$ is almost flat. Fig. $2 \mathrm{D}$ shows the results from an experiment performed under the same external magnetic field as shown in Fig. 2B but with the $\boldsymbol{H}_{\mathrm{IP}}$ rotated $90^{\circ}$ to align along the $X$ axis of the sample through tilt angles $\alpha=0^{\circ}$ and $\beta$ $=3^{\circ}$. The periodic magnetic contrast in the LUEM images now becomes visible almost immediately after time zero and reaches a maximum in FFT intensity at approximately 250 ps ( $T_{3}$ in Fig. 2D). After this point, the FFT intensity of the magnetic contrast steadily decreases in intensity. This can also be observed directly in the LUEM inset images shown in Fig. 2D. Analysis of the line profiles extracted from the LUEM images shows that under these conditions no reversal in magnetic Lorentz contrast is observed (vertical dashed lines in Fig. $2 \mathrm{E}$ serve as a guide to the eye). Comparing Fig. $2 \mathrm{~B}$ and $\mathrm{D}$, the inflection point for the leading edge of the FFT intensity of the transient magnetic contrast is shifted by approximately $\Delta t \sim$
100 ps towards shorter delays for $\boldsymbol{H}_{\text {IP }}$ aligned along the $X$ axis, as shown in Fig. 2B.

Taken together the results described above indicate that the laser-induced transient magnetic contrast stems from a mechanism including local demagnetization dynamics in response to the formation of a transient thermal grating in the magnetic thin film sample. The periodic demagnetization combined with the applied external field results in a canting of the local metastable magnetization direction and a damped precessing motion of magnetic moments. The full precession period for samples with $\boldsymbol{H}_{\mathbf{I P}}$ aligned along the $Y$ axis can be extracted as the temporal separation between two states of equal sign of the magnetic contrast. For the results presented in Fig. $2 \mathrm{~B}$, the precession frequency is $\sim 3.3 \mathrm{GHz}$, close to the reported frequencies for permalloy under similar experimental conditions. $^{33}$

\section{Discussion}

To describe the experimental observations, we propose a model outlined in Fig. 3(A-C). Before the arrival of the fs-laser pump, the magnetic moment $\boldsymbol{M}_{\mathbf{0}}$ (red arrow in Fig. 3A) is aligned along its equilibrium direction under the applied magnetic field $\boldsymbol{H}_{\text {ext }}$ (blue arrow in Fig. 3A). Upon the arrival of the laser pulse, the sample experiences rapid demagnetization into a state $\boldsymbol{M}^{*}$. As a consequence, the metastable equilibrium direction of the magnetization (red dashed line in Fig. 3B) cants by an angle $\theta$ towards $\boldsymbol{H}_{\text {ext }}$ and a few picoseconds later, the local magnetic moments $(\boldsymbol{M})$ begin to precess around the metastable magnetization direction (Fig. 3C). ${ }^{34}$ The threetemperature model $^{35,36}$ describes how the electronic subsystem is excited during the initial few hundreds of femtoseconds (the duration of the excitation pulse). The relaxation of the electronic subsystem results in an energy transfer to the spin and lattice subsystems. Here, we focus on the lattice temperature whose relevant time scale is comparable to that of the observed magnetic precession: a few picoseconds to hundreds of picoseconds, or even nanoseconds, after photoexcitation. The local temperature of the thin magnetic film is linearly dependent on the local laser fluence, as shown in Fig. 3D. The temperature dependence of the magnetization may be evaluated from Weiss molecular field theory ${ }^{37}$ as is shown in Fig. 3E. Furthermore, the degree of demagnetization will influence the canting angle of the metastable magnetization direction. The canting angle $(\theta)$, which also represents the initial precession angle of the magnetic moments, as a function of demagnetization, is provided in Fig. 3F. Excitation by means of a TG results in a spatially periodic laser fluence distribution across the sample, as shown in Fig. 3G. On a few ps time scale, such excitation will result in a spatially periodic thermal distribution, as shown in Fig. $3 \mathrm{H}$. The local lattice temperature influences the local magnetization with a resulting spatial distribution as shown in Fig. 3I. A change in local magnetization in the presence of a constant applied magnetic field will in turn affect the metastable direction of the magne- 

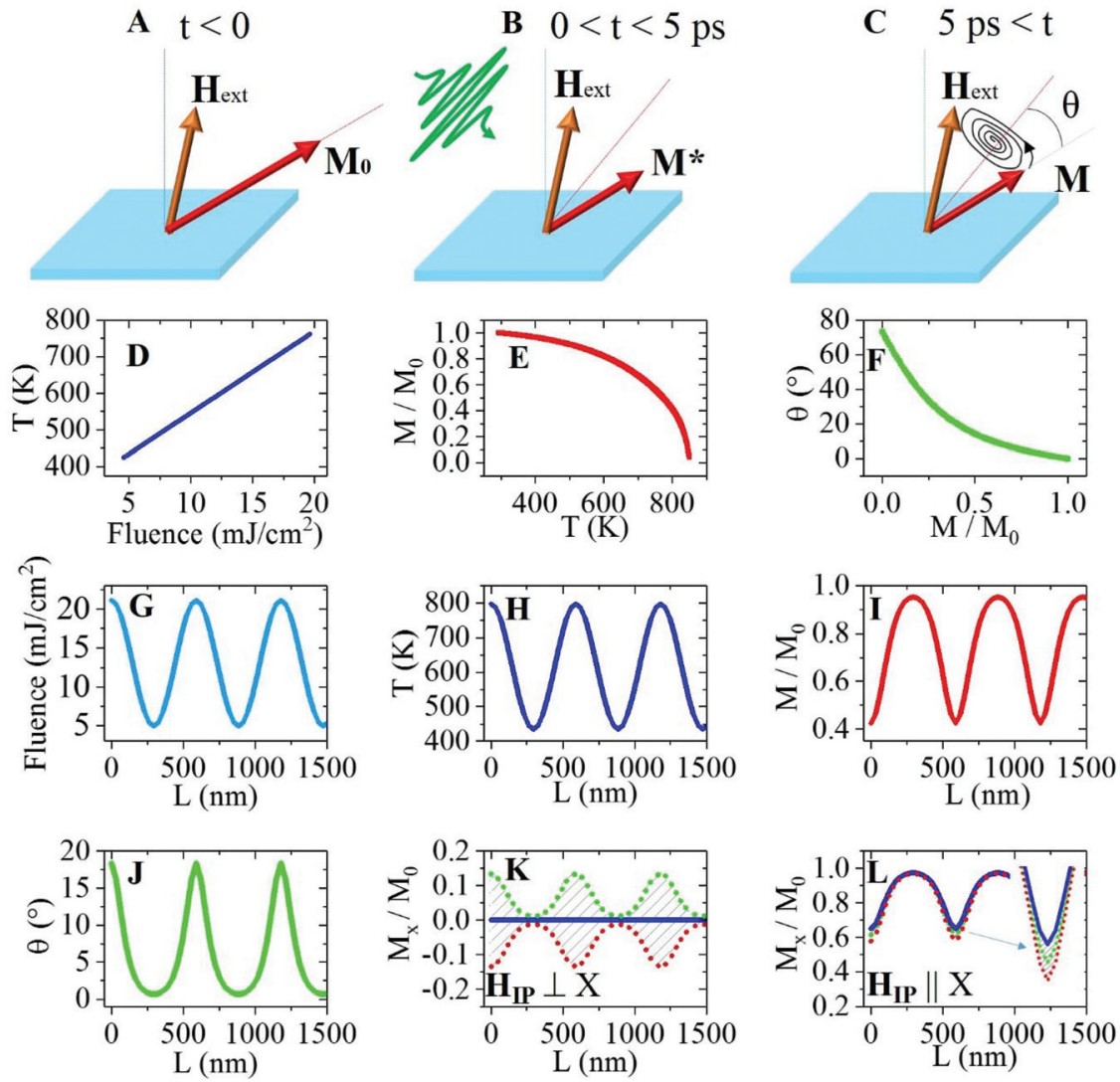

Fig. 3 Magnetic response to excitation by a TG. (A-C) Magnetization before, during, and after fs laser excitation. $M_{0}, M^{*}$, and $M$ represent the magnetic moment at $t<0,0<t<5 \mathrm{ps}$ and $t>5 \mathrm{ps}$. $H_{\text {ext }}$ is the external magnetic field. The red dashed line represents the metastable magnetization direction and $\theta$ is the precession angle. (D) Correlation of local laser fluence and local temperature. (E) Temperature dependence of the saturation magnetization of permalloy. (F) Initial precession angle, $\theta$, as a function of saturation magnetization in an external magnetic field $\mu_{0} H_{\text {ext }}=250 \mathrm{mT}$. Spatial distributions of laser fluence (G), sample temperature $(\mathrm{H})$, magnetization (I), and precession angle $(\mathrm{J})$ across the transient grating after photoexcitation. Spatial distributions of the $X$ components of the magnetization, $M_{X}$, with $H_{\mathbb{I P}}$ aligned along the $Y$ axis (K) and the $X$ axis (L). The blue lines represent the states of magnetization immediately after excitation. The green and red dotted lines in (K) indicate the magnetization at phase $\pi / 2$ and $3 \pi / 2$ subsequent to precessing motion of the magnetic moments. In (L), the corresponding dotted lines indicate phase $\pi / 2$ and $\pi$. The inset in (L) shows a magnified view of the local region with the largest demagnetization.

tization (effective field). From these results, we may estimate the initial local precession angle in Fig. 3J. The rapid change in metastable magnetization direction leads to a Larmor precession of the magnetic moments around the effective field direction as is schematically indicated in Fig. 3C. From the precession of the magnetic moments follows that the projection of the magnetization onto the $X$ direction changes with the phase of the precession. By applying the left-hand rule, for the Lorentz force on electrons, in the relevant experimental geometry we can conclude that only in-plane components of the magnetization will contribute to the Lorentz contrast. This means that only the in-plane magnetic component along the $X$ axis will contribute to the periodic Lorentz contrast in the $Y$ direction of the sample. Calculated in-plane $X$ components of the magnetization $\left(\boldsymbol{M}_{\boldsymbol{X}}\right)$, as a function of the spatial coordinate for $\boldsymbol{H}_{\mathrm{IP}}$ oriented along the $Y$-direction, is shown in Fig. $3 \mathrm{~K}$. At a temporal delay of a few picoseconds after photoexcitation, the sample is in a state of maximum demagnetization. However, the $\boldsymbol{M}_{\boldsymbol{X}}$ component is near zero since the precession of the magnetic moments has yet to begin (blue solid line in Fig. 3K). At longer delays, the precession will change the amplitude and direction of $\boldsymbol{M}_{\boldsymbol{X}}$ periodically in time within the range indicated by the red and green dotted traces. In contrast, when $\boldsymbol{H}_{\text {IP }}$ is oriented along the $X$ direction, the contribution to $\boldsymbol{M}_{\boldsymbol{X}}$ is dominated by the local rapid demagnetization (blue line in Fig. 3L). The variation in $\boldsymbol{M}_{\boldsymbol{X}}$ due to the precession of the magnetic moments is almost negligible, as shown in Fig. $3 \mathrm{~L}$ and its inset. Note that all spatial periodicities of the aforementioned parameters are the same and determined by the periodicity of the exciting optical grating. Details of the simulations in Fig. 3 (D-L) are presented in section S5.†

Based on the experimental results from Fig. 2 and the calculations presented in Fig. 3, we are now at the stage to describe the mechanism for the formation of the transient precessing magnetic grating. The illustration in Fig. 4A shows top views of the magnetization configuration at locations of constructive and destructive interference before time zero $(<0)$ and at selected phases/time delays $(0, \pi / 2, \pi$, and $3 \pi / 2)$ of the coher- 


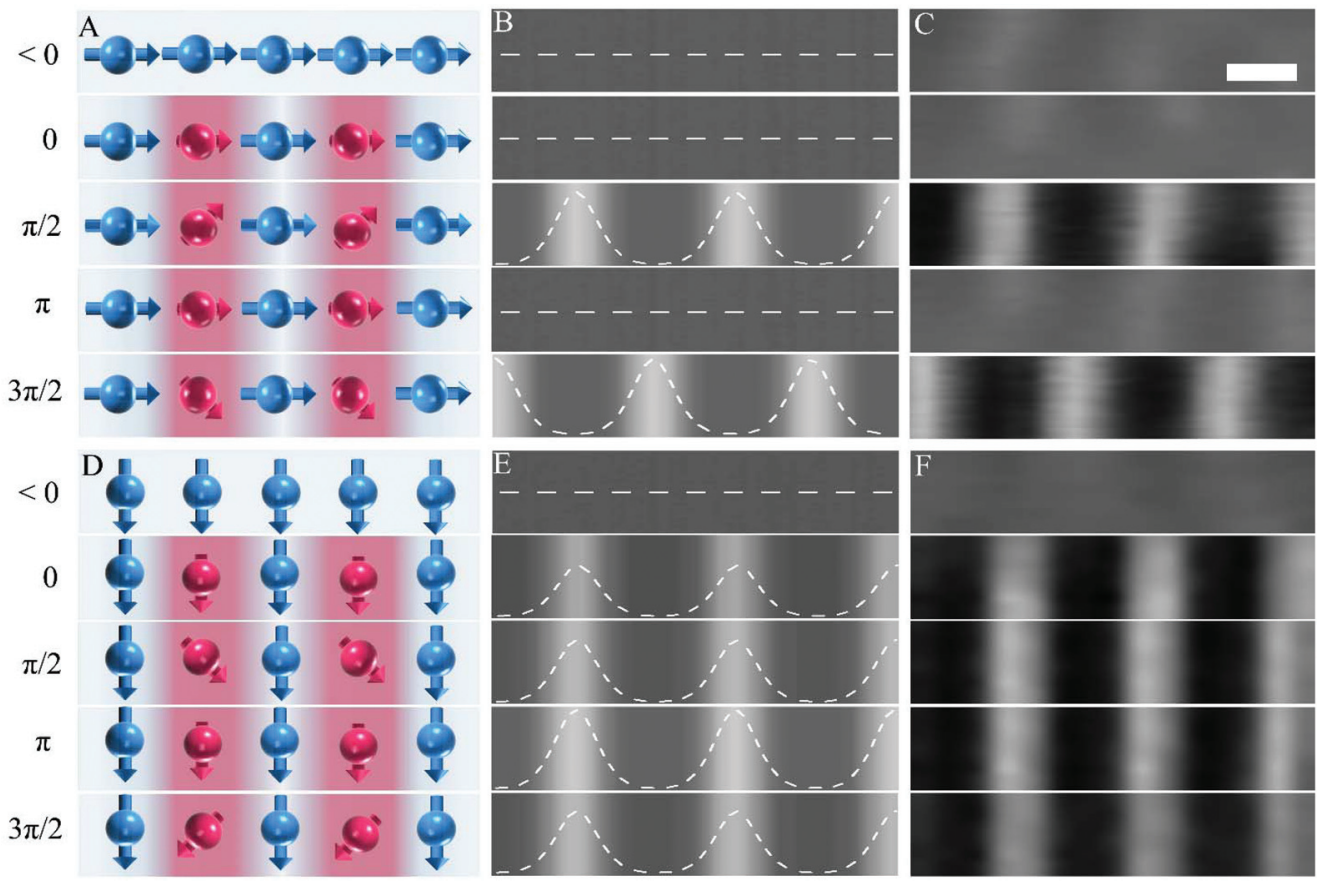

Fig. 4 Illustration of laser-induced magnetic precession and the resulting phase dependent Lorentz contrast. (A) Top views of the magnetization configurations with $H_{\mathrm{IP}}$ oriented along the $Y$ axis of the sample before time zero $(<0)$ and at different phases (time delays) of the precession: $0, \pi / 2, \pi$, and $3 \pi / 2$. The TG results in a transient precessing magnetic grating. Red shaded areas indicate regions with constructive interference (high local laser fluence), while light blue shaded regions indicate regions with destructive interference (low local laser fluence). (B) Simulated Lorentz TEM images from the corresponding magnetization configurations in (A). The white dashed lines show the intensity profiles of the simulated Lorentz contrast. (C) 2D-FFT filtered Lorentz images from experimental results of similar size as shown in the simulated images in (B). The scale bar in the upper panel corresponds to $250 \mathrm{~nm}$. (D) Top views of the magnetization configuration at different phases of the precession with $\mathrm{H}_{\mathrm{IP}}$ oriented along the $X$ direction of the sample. (E) Corresponding simulated Lorentz images and contrasts of (D). (F) 2D-FFT filtered Lorentz images from experimental results.

ent precession. The lengths of the vectors indicate the magnitude of the local magnetic moment. The laser pulse induced spatial variations of $\boldsymbol{M}_{\boldsymbol{X}}$ in the $Y$ direction, across the transient grating, determine the magnetic Lorenz contrast. Before time zero, the magnitude and direction of all magnetic moments are equal and aligned along the $Y$ direction, with no magnetic Lorentz contrast observed in the LUEM micrographs. Following excitation by the TG, the magnetic sample experiences a rapid periodic demagnetization. However, at short temporal delay (within a few picoseconds), all magnetic moments will essentially remain aligned along the $Y$ direction with negligible $\boldsymbol{M}_{\boldsymbol{X}}$ components. A configuration with all local magnetic moments aligned parallel to the magnetization gradient results in no magnetic Lorentz contrast. This is also the situation for precessing moments at phase 0 and $\pi$, which refer to the images at contrast minima $\left(T_{3}, T_{5}\right.$, and $T_{7}$ in Fig. 2B). In Fig. $3 \mathrm{~K}$, phase 0 and $\pi$ of the precession correspond to the blue line with zero $\boldsymbol{M}_{\boldsymbol{X}}$ components. $\boldsymbol{M}_{\boldsymbol{X}}$ will increase as the magnetic moments begin to precess around their metastable magnetization direction. Phase $\pi / 2$ in Fig. $4 \mathrm{~A}$ shows the precessing magnetic moments at a position for maximum $\boldsymbol{M}_{\boldsymbol{X}}$. This configuration corresponds to the upper green dotted line in Fig. $3 \mathrm{~K}$ and a maximum difference in $\boldsymbol{M}_{\boldsymbol{X}}$ (maximum magnetic Lorentz contrast) between the regions of constructive and destructive interference of the TG. The situation is similar for the precessing moments at phase $3 \pi / 2$, except for the reversal in the direction of $\boldsymbol{M}_{\boldsymbol{X}}$. The reversal of $\boldsymbol{M}_{\boldsymbol{X}}$ results in an inversion of the magnetic Lorentz image contrast compared to the situation with the precessing spins in phase $\pi / 2$, in excellent agreement with the experimental results presented in Fig. 2C. Fig. 4B shows the simulated Lorentz images for the magnetization configurations before zero $(<0)$ and in phases $0, \pi / 2, \pi$, and $3 \pi / 2$, which are referred to the inset images of $T_{0}-T_{5}$ in Fig. 2B. A description of the simulation can be found in section S6.† A direct comparison of the simulated Lorentz images and the experimental results (FFT filtered) from the same region of the sample can be seen in Fig. 4B and C. The agreement between simulation and experimental results shows that the physical model employed can describe the physics involved. Rotating the direction of $\boldsymbol{H}_{\mathrm{IP}}$ by $90^{\circ}$ to align parallel to the TG results in strikingly different magnetization dynamics. Fig. 4D shows the top views of the local magnetization configurations at different phases of the precession. The rapid demagnetization at time zero results in a configuration with all local magnetic moments aligned orthogonally to the local magnetization gradient. This configuration fulfills the conditions for magnetic Lorentz contrast and explains why the formation of magnetic contrast is sub- 
stantially faster compared to the configuration with the magnetic moments aligned parallel to the local magnetic gradient. The Lorentz contrast increases slightly as the magnetic precession proceeds to phase $\pi / 2$ and $\pi$. The increasing contrast may be traced from the change in $\boldsymbol{M}_{\boldsymbol{X}}$ at different phases of the precession as is shown in Fig. 3L. A maximum in Lorentz contrast is expected at phase $\pi$. This is in agreement with the experimentally observed maximum in the FFT intensity of the transient magnetic grating at $T_{3}$ in Fig. 2C. The expected modulation in Lorentz contrast by the precession of the magnetic moments is only about one tenth of the amplitude. This explains why no contrast inversion is observed. Simulated Lorentz images at selected phases of the precession are shown in Fig. 4E and as comparison FFT filtered Lorentz images from the same sample region are presented as shown in Fig. $4 \mathrm{~F}$. The process for establishing magnetic Lorentz contrast is distinctly different for the configurations with the magnetic moments aligned parallel and orthogonal to the local magnetic gradient. This explains the temporal shift in inflection points of the initial rising edges in magnetic contrast points, $\Delta t$, between Fig. $2 \mathrm{~B}$ and $\mathrm{D}$. In the configuration with the magnetic moments aligned parallel to the local magnetic gradient, no magnetic contrast is expected immediately after excitation and maximum contrast occurs after a quarter period $(\pi / 2)$ of the precession. This is unlike the situation for a configuration with the magnetic moments aligned orthogonally to the local magnetic gradient where the rapid demagnetization results in an almost immediate Lorentz contrast which is only moderately modulated by the precession of the magnetic moments. Taken together, the presented model describing a precessing transient magnetic grating explains the experimental results for the magnetic Lorentz contrast presented in Fig. 2B-E.
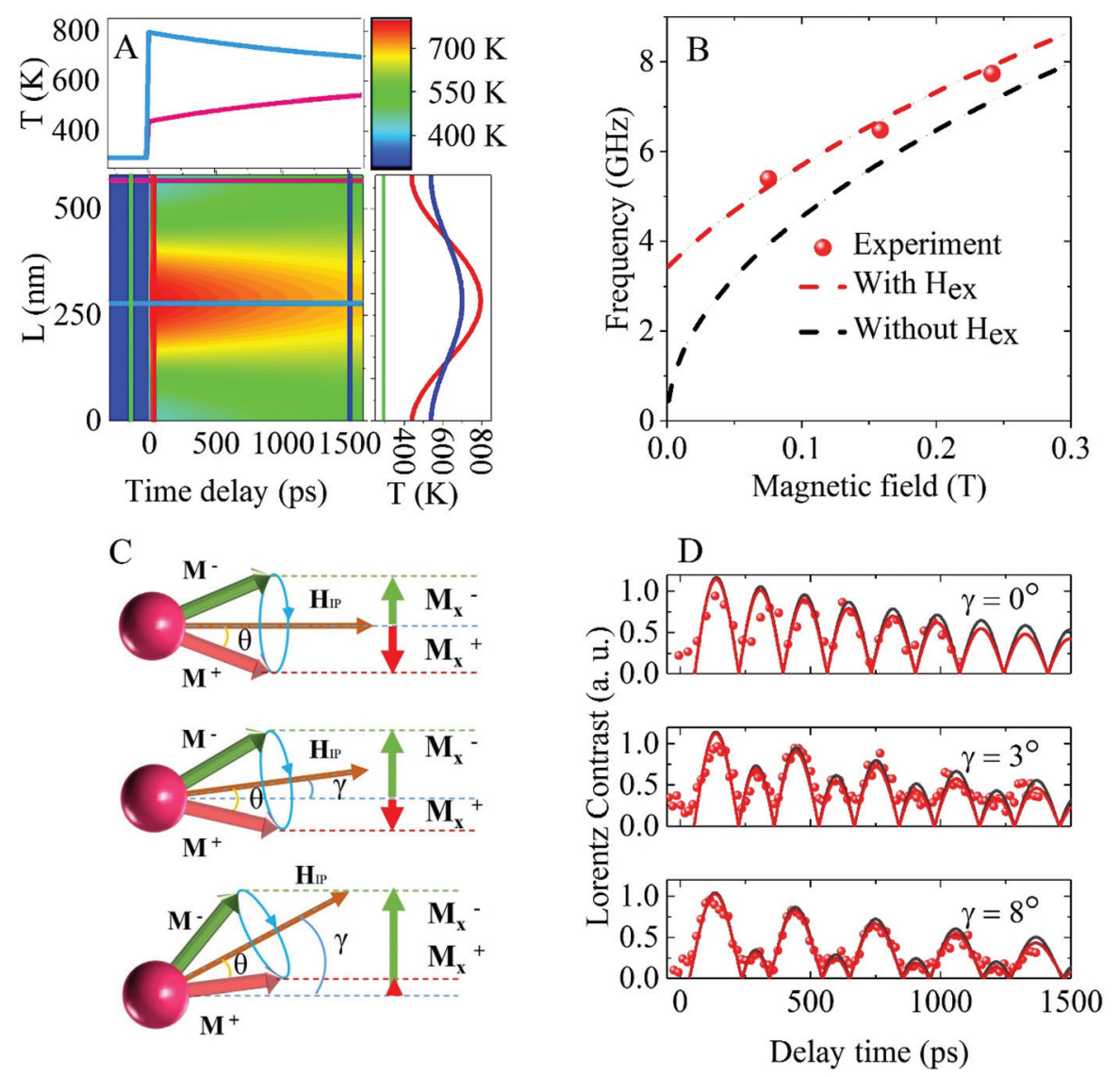

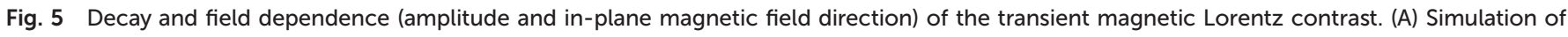

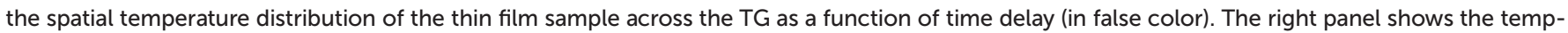

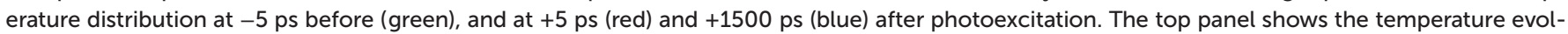

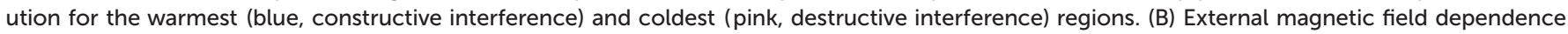

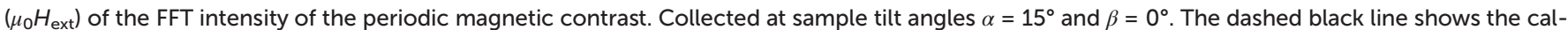

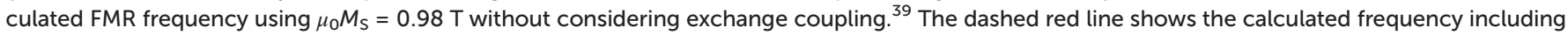

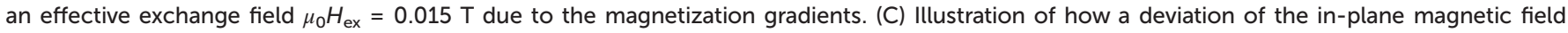

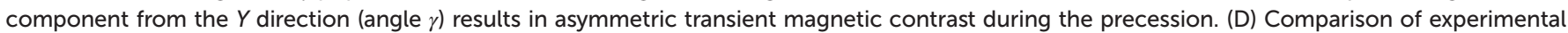

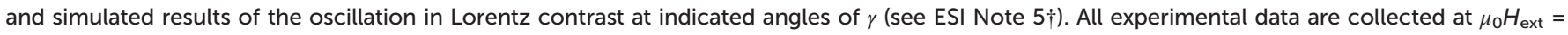

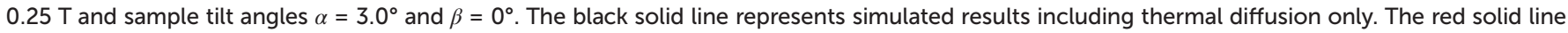
represents simulated results including both thermal diffusion and Gilbert damping. 
We now focus our attention to the decay factors of the magnetic gratings governing the time dependent decay in magnetic Lorentz contrast. Two decay processes are considered: (1) dissipation of the spatial thermal gratings ${ }^{39}$ and (2) Gilbert damping of the precession through energy transfer to the phonon subsystem. We begin by simulating the local temperature distribution as a function of time delay, as shown in Fig. 5A and section S4. $\dagger$ Thermal conduction will reduce the temperature difference between regions of constructive (hot) and destructive (cold) optical interference. The temperature difference decreases from approximately $360 \mathrm{~K}$ to about $160 \mathrm{~K}$ within 1.5 ns. From diminishing thermal gradients a reduction in magnetization gradients and magnetic Lorentz contrast is followed. The second decay process is due to Gilbert damping of the precession angle of the magnetic moments. Gilbert damping drags the precession of the magnetic moments closer to the effective field direction (red dashed line in Fig. 3C). A combination of both these effects is considered for the fit of the experimental LUEM results shown in the upper panel of Fig. 5D. The effective damping for permalloy exhibits a weak temperature dependence under the relevant experimental conditions. ${ }^{40}$ Therefore, we use the room temperature Gilbert damping parameter $\left(\alpha_{\text {eff }}=0.008\right)$ in the simulations. ${ }^{40}$ The thermal diffusion only (black solid line) and combined thermal and Gilbert damping (red solid line) contribution to the decay in Lorentz image contrast is shown in the upper panel of Fig. 5D. A comparison of the simulated thermal and the combined thermal and Gilbert damping results shows that thermal diffusion dominates the decay in Lorentz image contrast at the current periodicity of the transient grating, but a better fit to the experimental results is found after including the contribution from the Gilbert damping.

The precession frequency of the magnetic moments depends on the external magnetic field. ${ }^{33}$ The oscillation frequency of the FFT intensity of the periodic magnetic Lorentz contrast represents a direct measure of the precession frequency. Fig. 5B shows the oscillation frequency of the magnetic contrast as a function of the applied magnetic field. The experimental results were extracted at a relatively high tilt angle of the sample $\left(\alpha=15^{\circ}\right.$ and $\left.\beta=0^{\circ}\right)$ in order to allow for several oscillation periods within a few ns temporal delay. The precession frequency increases with the external field following the expected trend from simulated ferromagnetic resonance (FMR, black dashed line) frequency. ${ }^{38}$ The discrepancy between the experimental and calculated results may tentatively be attributed to the inhomogeneous demagnetization (shown in Fig. 3I) and the different precession angles (shown in Fig. 3J) in the sample, which may result in a significant exchange coupling. An additional effective field $H_{\mathrm{ex}}$ was included in the frequency calculations to accommodate for the contribution from the exchange coupling (see section $\mathrm{S} 5 \dagger$ ). The calculated frequency (red dashed line in Fig. 5B) now shows a good agreement with the experimental results.

A deviation of the in-plane magnetic field from the $Y$ direction is expected to influence the transient magnetic Lorentz contrast during the precession. Precession at three different angles of $\gamma$ (where $\gamma$ is defined as the angle between $\boldsymbol{H}_{\text {IP }}$ and $Y$ ) is illustrated in Fig. 5C. In the case of $\gamma=0$ (upper panel), the absolute $\boldsymbol{M}_{\boldsymbol{X}}$ projections and the resulting FFT intensities of the Lorentz contrast will be similar at phase $\pi / 2$ and $3 \pi / 2$. Under the conditions $0<\gamma<\theta$, the FFT intensity will be asymmetric during the precession period and the peaks at the $\pi / 2$ and $3 \pi / 2$ phase may be discriminated by their relative amplitude as $\gamma$ and $\theta$ determines the relative amplitude of the peaks. Fig. 5D shows the matching experimental results and simulations of the absolute Lorentz image contrast for $\gamma$ at $0^{\circ}, 3^{\circ}$ and $8^{\circ}$ and at an initial precession angle $\theta=17^{\circ}$. Under the conditions $\theta \leq \gamma<\pi / 2$, the $\mathbf{M}_{\mathbf{X}}$ projection will not change sign during the precession and no reversal of Lorentz contrast can be detected. Further theoretical studies will be helpful in providing a comprehensive understanding to the damping mechanisms involved at the nanoscale in these transient magnetic gratings.

\section{Conclusions}

To summarize, we have demonstrated that the combination of TG and LUEM facilitates imaging of local magnetic dynamics at combined high spatial (nm) and temporal (ps) resolutions. Excitation via a TG induces an ultrafast periodic demagnetization of the sample with a resulting formation of a transient precessing magnetic grating. The transient magnetization dynamics is found to be sensitive to the alignment of the inplane magnetic field $\left(\mathbf{H}_{\mathbf{I P}}\right)$ with respect to the optical grating. Fields aligned orthogonal to the direction of the optical grating result in strong oscillations of the magnetic Lorentz contrast, while parallel fields show only limited oscillation. The experimental results can be described by a model including the phase of the precessing magnetic moments. From the time-resolved LUEM results, we extract detailed information of the magnetic precession, such as local magnetization, precession frequency and magnetic field dependence, and relevant decay factors of the magnetic gratings.

The application of TG-induced magnetic gratings in LUEM may tentatively be extended to the studies of spin waves as it is sensitive to the local phase of the precession and may be deployed under external field, critical in studies of spin wave propagation. Also, excitation by means of TG offers the potential for describing the mechanisms determining magnetization dynamics on its intrinsic time and length scales. The periodicity of the transient magnetic grating can readily be tuned by the laser wavelength or the angle between the two incoming laser beams. A natural extension of the method would be excitation through extreme ultraviolet (EUV) wavelengths, facilitating optical gratings on a few nanometer scale. ${ }^{41,42}$ The TG facilitates the formation of magnetic domains with tunable nanoscale dimensions, which may allow the study of magnetic dynamics at dimensions comparable to the intrinsic scales of domain walls or other magnetic structures, such as vortices or skyrmions. Furthermore, analysis of local temperatures and thermal diffusion at combined nanometer and picosecond res- 
olutions in materials and devices is challenging. ${ }^{43}$ As demonstrated in this paper, the transient magnetic contrast in LUEM may serve as a sensitive probe of the transient temperature distribution of magnetic samples and may allow mapping of the local temperature and ultrafast thermal diffusion processes. In other words, the combination of LUEM with TG can be used as an ultrafast nano-thermometer. Finally, we also envision how this technique may be extended to the studies of dynamics in other classes of magnetic materials, such as ferrimagnets or antiferromagnets. As the image contrast presented here originates from the magnetic gradients induced by TG or thermal gratings, it may be feasible to induce sufficient magnetic gradients in systems not necessarily limited in ferromagnets.

\section{Materials and methods}

\section{Sample preparation}

$50 \mathrm{~nm}$ thick permalloy $\left(\mathrm{Ni}_{80} \mathrm{Fe}_{20}\right)$ thin films were sputtered on $50 \mathrm{~nm}$ thick $\mathrm{Si}_{3} \mathrm{~N}_{4}$ electron transparent membrane grids for transmission electron microscopy analysis (Ted Pella, USA). The samples were positioned with the slanted side faces facing up, ensuring metallization of both the membrane and side faces. The magnetron sputtering deposition was performed in a high-vacuum chamber with a base pressure of less than $3 \times$ $10^{-8}$ Torr [AJA Orion 8]. The argon working pressure was 2.5 mTorr during deposition. All samples were capped with a $3 \mathrm{~nm}$ thick $\mathrm{Al}$ film to prevent oxidation of the $\mathrm{Ni}_{80} \mathrm{Fe}_{20}$.

\section{Sample geometry}

The sample was placed on the TEM sample holder with the slanted faces of the grid facing towards the direction of the incident electron beam, as indicated in Fig. 2A. The angle of the slanted faces relative to the membrane normal was $\varphi=$ $35.26^{\circ}$. The thickness of the Si grid frame was $200 \mu \mathrm{m}$, the thickness of the $\mathrm{Si}_{3} \mathrm{~N}_{4}$ membrane was $50 \mathrm{~nm}$, and the membrane window size was $100 \mu \mathrm{m} \times 1500 \mu \mathrm{m}$. The long edge of the window was carefully positioned parallel to the $X$ axis of the double-tilt TEM holder. The tilt angles $\alpha$ and $\beta$ are defined as positive for anti-clockwise rotation around the $X$ and $Y$ axis, as shown in Fig. 2A. Fig. 2A illustrates the geometry for the laser illumination of the sample. The laser was carefully aligned to illuminate the intersection between the slanted faces and the membrane surface. The permalloy thin film on the side faces allows them to serve as reflecting mirrors, with approximately $50 \%$ reflectivity for $515 \mathrm{~nm}$ wavelength at an incident angle of $45^{\circ}$. The interference of the direct and reflected laser beams results in the formation of a TG at the membrane surface.

\section{Lorentz mode ultrafast electron microscopy (LUEM)}

To avoid saturation of the magnetization of the soft permalloy sample, the ultrafast electron microscope may be operated with the objective lens switched off. Under such operational conditions, the residual magnetic field at the sample position may be kept below 10 Oe and allows for Lorentz mode imaging of the magnetization configuration in the sample. The external magnetic field can be controlled by applying a current to the objective lens. The magnetic field at the sample position was calibrated for relevant objective lens currents by means of a Hall probe TEM holder. The direction of the applied magnetic field is perpendicular to the sample surface at zero sample tilt angle. An in-plane component of the external magnetic field may be realized through the application of $\alpha$ or $\beta$ tilt angles (see Fig. 2A).

The sample was excited by $300 \mathrm{fs}$ laser pulses $(\lambda=515 \mathrm{~nm}, f$ $=35 \mathrm{kHz}$ ) focused to a $135 \mu \mathrm{m}$ diameter Gaussian spot at a

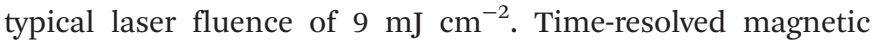
information was recorded by electron bunches generated through photoemission from a $\mathrm{LaB}_{6}$ guard ring cathode by $\mathrm{UV}$ laser pulses $(\lambda=258 \mathrm{~nm})$ from the same laser source as the excitation pulse. The relative time delay between the pump and probe pulses was tuned by means of a motorized delay stage that allows for varying the optical path length for the pump pulses. Further details of the LUEM setup can be found in ref. 43. The temporal pulse length of the electron bunches was determined by means of photo-induced near field electron microscopy using a Gatan Quantum SE electron energy analyzed. Under the imaging conditions relevant for the present study, the electron pulse length was estimated to be approximately 2 ps. The ultrafast Lorentz micrographs were recorded using a CheeTah1800 (a Medipix3Rx based camera from Amsterdam Scientific Instruments, the Netherlands).

\section{Funding}

This work was supported by the Knut and Alice Wallenberg Foundation, the Swedish Research Council (VR), and partially by the Horizon 2020 research and innovation programme (ERC Advanced Grant No. 835068 "TOPSPIN").

\section{Author contributions}

G. C. and S. J. performed the sample growth and experimental analysis. J. A and J. W. coordinated the project. All the authors analyzed the results and co-wrote the manuscript. The authors thank Max Yan for fruitful discussions.

\section{Data and materials availability}

The data that support the plots within this paper and other findings of this study are available from the corresponding author upon reasonable request.

\section{Conflicts of interest}

There are no conflicts to declare. 


\section{Notes and references}

1 C. Högemann, M. Pauchard and E. Vauthey, Picosecond transient grating spectroscopy: The nature of the diffracted spectrum, Rev. Sci. Instrum., 1996, 67, 3449-3453.

2 F. Hofmann, M. P. Short and C. A. Dennett, Transient grating spectroscopy: An ultrarapid, nondestructive materials evaluation technique, MRS Bull., 2019, 44, 392402.

3 A. A. Maznev, et al., Generation of coherent phonons by coherent extreme ultraviolet radiation in a transient grating experiment, Appl. Phys. Lett., 2018, 113, 221905.

4 J. A. Johnson, et al., Direct measurement of room-temperature nondiffusive thermal transport over micron distances in a silicon membrane, Phys. Rev. Lett., 2013, 110, 025901.

5 F. Bencivenga, et al., Nanoscale transient gratings excited and probed by extreme ultraviolet femtosecond pulses, Sci. $A d v .$, 2019, 5, eaaw5805.

6 M. Fuchs, et al., Anomalous nonlinear X-ray Compton scattering, Nat. Phys., 2015, 11, 964-970.

7 T. E. Glover, et al., X-ray and optical wave mixing, Nature, 2012, 488, 603-608.

8 F. Bencivenga, et al., Four-wave mixing experiments with extreme ultraviolet transient gratings, Nature, 2015, 520, 205-208.

9 R. K. Lam, et al., Soft X-Ray Second Harmonic Generation as an Interfacial Probe, Phys. Rev. Lett., 2018, 120, 023901.

10 J. Goldshteyn, A. Bojahr, P. Gaal, D. Schick and M. Bargheer, Selective preparation and detection of phonon polariton wavepackets by stimulated Raman scattering, Phys. Status Solidi B, 2014, 251, 821-828.

11 N. Gedik, J. Orenstein, R. Liang, D. A. Bonn and W. N. Hardy, Diffusion of Nonequilibrium Quasi-Particles in a Cuprate Superconductor, Science, 2003, 300, 1411.

12 S. Tang, M. Wang and B. D. Olsen, Anomalous SelfDiffusion and Sticky Rouse Dynamics in Associative Protein Hydrogels, J. Am. Chem. Soc., 2015, 137, 3946-3957.

13 N. K. Ravichandran, H. Zhang and A. J. Minnich, Spectrally Resolved Specular Reflections of Thermal Phonons from Atomically Rough Surfaces, Phys. Rev. X, 2018, 8, 041004.

14 N. Osada, et al., Photoexcited carrier dynamics of doublelayered CdS/CdSe quantum dot sensitized solar cells measured by heterodyne transient grating and transient absorption methods, Phys. Chem. Chem. Phys., 2014, 16, 5774-5778.

15 A. Leblanc, et al., Plasma holograms for ultrahigh-intensity optics, Nat. Phys., 2017, 13, 440-443.

16 J. Janušonis, et al., Transient Grating Spectroscopy in Magnetic Thin Films: Simultaneous Detection of Elastic and Magnetic Dynamics, Sci. Rep., 2016, 6, 29143.

17 X.-G. Wang, L. Chotorlishvili and J. Berakdar, Strain and Thermally Induced Magnetic Dynamics and Spin Current in Magnetic Insulators Subject to Transient Optical Grating, Front. Mater., 2017, 4, 19.

18 C. S. Davies, et al., Towards massively parallelized alloptical magnetic recording, J. Appl. Phys., 2018, 123, 213904.
19 A. Kirilyuk, A. V. Kimel and T. Rasing, Ultrafast optical manipulation of magnetic order, Rev. Mod. Phys., 2010, 82, 2731-2784.

20 M. van Kampen, et al., All-optical probe of coherent spin waves, Phys. Rev. Lett., 2002, 88, 227201.

21 X. Yu, Y. Onose, N. Kanazawa, et al., Real-space observation of a two-dimensional skyrmion crystal, Nature, 2010, 465, 901-904.

22 H. S. Park, J. S. Baskin and A. H. Zewail, 4D Lorentz electron microscopy imaging: Magnetic domain wall nucleation, reversal, and wave velocity, Nano Lett., 2010, 10, 37963803.

23 A. Arbouet, G. M. Caruso and F. Houdellier, Ultrafast Transmission Electron Microscopy: Historical Development, Instrumentation, and Applications, Adv. Imaging Electron Phys., 2018, 207, 1-72.

24 S. A. Aseyev, E. A. Ryabov, B. N. Mironov and A. A. Ischenko, The development of ultrafast electron microscopy, Crystals, 2020, 10, 3390.

25 K. B. Schliep, P. Quarterman, W. Jian-Ping and D. J. Flannigan, Picosecond Fresnel transmission electron microscopy, Appl. Phys. Lett., 2017, 110, 222404.

26 G. Berruto, et al., Laser-Induced Skyrmion Writing and Erasing in an Ultrafast Cryo-Lorentz Transmission Electron Microscope, Phys. Rev. Lett., 2018, 120, 117201.

27 N. Rubiano da Silva, et al., Nanoscale Mapping of Ultrafast Magnetization Dynamics with Femtosecond Lorentz Microscopy, Phys. Rev. X, 2018, 8, 031052.

28 M. Zhang, et al., High Spatiotemporal Resolution of Magnetic Dynamics in Mn-Ni-Ga via Four-Dimensional Lorentz Microscopy, Phys. Rev. Appl., 2019, 12, 034037.

29 M. Möller, J. H. Gaida, S. Schäfer and C. Ropers, Few-nm tracking of current-driven magnetic vortex orbits using ultrafast Lorentz microscopy, Commun. Phys., 2020, 3, 1038.

30 M. Stärk, et al., Controlling the magnetic structure of $\mathrm{Co} /$ $\mathrm{Pd}$ thin films by direct laser interference patterning, Nanotechnology, 2015, 26, 20.

31 J. Janušonis, et al., Ultrafast magnetoelastic probing of surface acoustic transients, Phys. Rev. B, 2016, 94, 024415.

32 C. S. Davies, et al., Towards massively parallelized alloptical magnetic recording, J. Appl. Phys., 2018, 123, 213904.

33 C. Kittel, On the Gyromagnetic Ratio and Spectroscopic Splitting Factor of Ferromagnetic Substances, Phys. Rev., 1949, 76, 743.

34 A. A. Rzhevsky, B. B. Krichevtsov, D. E. Bürgler and C. M. Schneider, Magnetization dynamics induced by ultrashort optical pulses in $\mathrm{Fe} / \mathrm{Cr}$ thin films, Phys. Rev. B: Condens. Matter Mater. Phys., 2007, 75, 224434.

35 L. Perfetti, et al., Ultrafast electron relaxation in superconducting $\mathrm{Bi}_{2} \mathrm{Sr}_{2} \mathrm{CaCu}_{2} \mathrm{O}_{8+\delta}$ by time-resolved photoelectron spectroscopy, Phys. Rev. Lett., 2007, 99, 197001.

36 J. Walowski, et al., Energy Equilibration Processes of Electrons, Magnons, and Phonons at the Femtosecond Time Scale, Phys. Rev. Lett., 2008, 101, 237401. 
37 C. Kittel, et al., Introduction to Solid State Physics, John Wiley \& Sons, 8th edn, 2004. ISBN 978-0-471-41526-8.

38 S. Jiang, et al., Reduced spin torque nano-oscillator linewidth using $\mathrm{He}^{+}$irradiation, Appl. Phys. Lett., 2020, 116, 072403.

39 J. Janušonis, et al., Transient Grating Spectroscopy in Magnetic Thin Films: Simultaneous Detection of Elastic and Magnetic Dynamics, Sci. Rep., 2016, 6, 29143.

40 M. Shigemi, et al., Gilbert Damping for Various $\mathrm{Ni}_{80} \mathrm{Fe}_{20}$ Thin Films Investigated Using All-Optical Pump-Probe
Detection and Ferromagnetic Resonance, Appl. Phys. Express, 2008, 1, 121301.

41 F. Bencivenga, et al., Four-wave mixing experiments with extreme ultraviolet transient gratings, Nature, 2015, 520, 205.

42 F. Bencivenga, et al., Nanoscale transient gratings excited and probed by extreme ultraviolet femtosecond pulses, Sci. Adv., 2019, 5, 5805.

$43 \mathrm{~J}$. Shaozheng, et al., Influence of cathode geometry on electron dynamics in an ultrafast electron microscope, Struct. Dyn., 2017, 4, 054303. 